\title{
La culture entrepreneuriale : le quoi, le pourquoi et le comment
}

\author{
Paul-Arthur Fortin ${ }^{1}$
}

En 1985, le quotidien Le Soleil a publié pendant seize semaines, à raison de deux pages par semaine, le contenu du cours-concours Devenez entrepreneur qui a connu un franc succès. Celui-ci a été repris dans toutes les régions du Québec grâce à la complicité des principaux quotidiens. Finalement, l'essentiel du contenu d'alors a été publié dans le volume Devenez entrepreneur avec, comme sous-titre lors de la première édition, Pour un Québec plus entrepreneurial ${ }^{2}$.

Naïvement, je faisais alors l'hypothèse que la venue de nouveaux entrepreneurs amènerait dans son sillage la culture entrepreneuriale au Québec. Après 18 ans d'observation et de recherches, il convient de modifier cette proposition. Celle-ci deviendrait alors: La culture entrepreneuriale, pour plus d'entrepreneurs au Québec.

En effet, si une direction causale devait exister entre la culture entrepreneuriale et le niveau d'entrepreneurship, elle irait dans le sens de la culture à l'entrepreneurship et non l'inverse.

À bien y penser, la nature nous fournit moult exemples d'une relation semblable, que ce soit entre la mer et le poisson qui y habite, entre la terre et les récoltes qu'on en obtient ou entre l'arbre et les fruits qu'il porte. Pourquoi en serait-il autrement lorsqu'il s'agit des entrepreneurs et de la société qui les suscite?

Maintenant c'est clair. Une société qui désire plus d'entrepreneurs pour assurer sa survie et sa croissance à long terme doit travailler à développer d'abord la culture entrepreneuriale dans son milieu. S'il fallait une raison pour justifier la culture entrepreneuriale, en voilà une bonne et ce n'est pas la seule. Comme on le verra plus loin, la culture entrepreneuriale contribue aussi à une plus grande création de richesse.

Lorsqu'on parle de culture, on parle d'un concept vague, difficile à cerner dans le temps et à circonscrire dans l'espace. Pourtant, la culture fait toute la différence. Voyons de quoi il s'agit.

Dans le numéro spécial du journal Les Affaires portant sur le $75^{\mathrm{e}}$ anniversaire et publié en février 2003, deux articles méritent d'être signalés, facilitant ainsi la compréhension du concept en question.

En page 101, dans un article signé par Danielle Turgeon, on titre : «Lors de la création des HEC en 1907, plusieurs personnes s'insurgeaient parce qu'on allait former des voleurs $»$.

En page 59 du même journal, Pierre Théroux écrit : «Par ailleurs, l'Église continue d'avoir un ascendant idéologique important » et il cite Mgr Louis Adolphe Paquet, l'un des plus éminents théologiens du Canada français, qui déclarait en 1902: «Notre mission est moins de manier des capitaux que de remuer des idées, elle consiste moins à allumer le feu des usines qu'à entretenir et à faire rayonner au loin le foyer de la religion et de la pensée face à la montée de l'industrialisation $»$.

Dans l'histoire du Québec, des centaines d'autres anecdotes de ce type peuvent être racontées, certaines sont particulières à des régions, à des localités, voire même à des familles. Bien sûr, vous répondrez qu'il s'agit là de vieilles histoires que tout le monde a oubliées. En êtes-vous si sûr? Pour vous convaincre du contraire, voyez cette anecdote de lecture qui, je pense, explique bien comment se construit une culture : 
Mettez cinq chimpanzés dans une chambre...

Accrochez une banane au plafond et mettez une échelle permettant d'y accéder.

Assurez-vous qu'il n'y a pas d'autres moyens d'attraper la banane que d'utiliser l'échelle.

Mettez en place un système qui fait tomber de l'eau glacée dans toute la chambre dès qu'on commence à escalader l'échelle. Les chimpanzés apprennent vite qu'il ne faut pas escalader l'échelle.

Arrêtez le système d'eau glacée pour que l'escalade n'ait plus son effet gelé.

Maintenant, remplacez l'un des chimpanzés par un nouveau. Ce dernier va chercher à escalader l'échelle. Sans comprendre pourquoi, il se fera tabasser par les autres.

Remplacez encore un des vieux chimpanzés par un nouveau. Il se fera encore tabasser. C'est le chimpanzé $n^{\circ} 6$, le dernier entré, qui tapera le plus fort.

Continuez le processus jusqu'à ce qu'il ne reste que des nouveaux chimpanzés. Aucun ne cherchera à escalader l'échelle. Si, pour une raison ou une autre, l'un deux ose y penser, il se fera massacrer illico presto par les autres. Le pire, c'est qu'aucun des chimpanzés n'a la moindre idée sur le pourquoi de la chose. C'est ainsi que la culture prend naissance. ${ }^{3}$

Dans de telles circonstances, vous admettrez qu'il n'est pas facile de saisir la culture et de la comprendre.

Vouloir changer la culture d'une société, cela est presque impossible à court terme, à moins d'une crise importante ou d'un effort concerté de plusieurs acteurs importants de la société. Ce que je propose dans mon volume sur La culture entrepreneuriale, un antidote à la pauvreté ${ }^{4}$, c'est de développer une culture nouvelle, la culture entrepreneuriale, en mobilisant toutes les ressources de bonne volonté. C'est là une façon d'éviter des luttes stériles avec des fantômes du passé.

Voici ma définition de la culture entrepreneuriale adaptée, je crois, à la situation québécoise : une forte croyance en dix vérités qui incitent les milieux soucieux de leur survie et de leur développement à prioriser l'entrepreneurship local et à se donner progressivement les moyens d'actualiser ce potentiel.

\section{Vouloir changer la culture d'une société, cela est presque impossible à court terme, à moins d'une crise importante ou d'un effort concerté de plusieurs acteurs importants de la société.}

\section{Les dix vérités}

1. Le développement passe d'abord par des personnes, notamment des leaders locaux, qui facilitent par leur animation et leurs initiatives l'émergence d'une communauté qui veut s'assumer économiquement. Les structures ou les programmes suivent ensuite.

2. L'entrepreneur crée l'entreprise. Encore là, celuici peut être féminin ou masculin, individuel ou collectif. L'entrepreneur, c'est une personne habile à transformer un rêve, un problème ou une opportunité en une entreprise viable. Dit autrement par une entrepreneure de Madagascar, «c'est un importateur d'angoisses et un exportateur de dynamisme ». L'entrepreneur, c'est un cadeau pour une société.

3. L'entrepreneur, c'est le fruit de son milieu. Un milieu qui n'aime pas les entrepreneurs et qui n'en veut pas a de bonnes chances d'être exaucé. Par ailleurs, si, au contraire, on en désire comme dans le sport, il devient alors utile d'établir une complicité entre la famille, l'école, la cité et les entreprises existantes pour développer un terreau fertile, un milieu incubateur d'entrepreneurship, à terme, une véritable culture entrepreneuriale.

4. On devient entrepreneur, d'où l'importance de la formation et de l'apprentissage. La culture entrepreneuriale peut difficilement se développer dans un milieu sans la contribution de l'école, tant au plan des attitudes et des valeurs que des connaissances et des compétences. L'école demeure le moyen clé pour découvrir le potentiel entrepreneurial, le soutenir et l'actualiser.

5. Le potentiel entrepreneurial existe dans nos milieux pour créer les entreprises et les emplois nécessaires. Bien sûr, ce potentiel, pour s'épanouir correctement, nécessite un milieu favorable à l'entrepreneurship comme on l'a indiqué plus 
haut. Les études du GEM ${ }^{5}$ confirment d'année en année cette vérité de l'existence de cette capacité d'entreprendre partout sur la planète. L'étude de GEM (2002) porte sur 37 pays réunissant $62 \%$ de la population mondiale et produisant $92 \%$ du PIB de la planète. Dans cette recherche, on compare le pourcentage des personnes de 18 à 64 ans travaillant à créer une entreprise ou gérant une entreprise de 42 mois et moins. En 2002, le pourcentage moyen dans les 37 pays était de $12 \%$ et de $10,5 \%$ pour les États-Unis. On n'a pas l'information pour le Québec en 2002, du moins pas encore. Mais pour 1999, 2000 et 2001 , le pourcentage moyen était de $5,9 \%$ au Québec comparativement à $6,6 \%$ au Canada, selon une analyse de Nathaly Riverin ${ }^{6}$. Fort de ces recherches, la question devient alors : pourquoi le potentiel entrepreneurial serait-il différent au Québec ? Pourquoi serait-il différent au Saguenay-Lac Saint-Jean ou en Beauce ? Si l'on fait abstraction de l'effet de la culture sur le comportement, les recherches effectuées par le GEM nous autorisent à conclure que le potentiel entrepreneurial est réparti également sur toute la planète. La moisson dépend de l'engagement du milieu à croire en son développement et à l'organiser.

6. L'entrepreneurship, c'est un outil incontournable pour les gens pauvres qui veulent s'en sortir. On n'est jamais trop pauvre pour entreprendre, c'est juste un peu plus long. Attendre d'être riche pour entreprendre, ça risque de ne jamais arriver. Entreprendre pour devenir riche et éventuellement investir, c'est là la voie que des milliers d'entrepreneurs à succès ont choisie et continuent de choisir.

7. La créativité des hommes et des femmes est illimitée, assurant ainsi des réponses toujours plus innovatrices les unes que les autres pour adresser les innombrables besoins humains. Il n'y a pas de limites au nombre et à la diversité d'entreprises à créer pour répondre aux besoins humains.

8. Les besoins humains sont illimités en diversité. Il n'arrivera jamais qu'il n'y ait plus de besoins à satisfaire. C'est là une excellente nouvelle pour les entrepreneurs.
9. L'entreprise crée la richesse et l'emploi. Bien sûr, l'entreprise peut être sociale, économique ou culturelle. Elle peut être à but lucratif ou non, coopérative, individuelle ou de groupe. Ce qu'il faut retenir ici, dans cette période de besoin de revenus grandissants de l'État pour assumer ses obligations, notamment en santé et en éducation, c'est que le niveau de création de richesses et de rentrées fiscales peut varier selon la nature des entreprises. Règle générale, l'entreprise issue du milieu et enracinée dans le milieu assure une meilleure stabilité que la filiale ou la succursale d'une entreprise étrangère, et les retombées économiques et sociales pourront varier grandement dans le temps. Il en est de même pour les entreprises sans but lucratif par rapport à des entreprises à but lucratif. Bref, dans une période où la ressource humaine disponible diminue, il faut être plus sélectif sur la nature des entreprises qu'on souhaite accueillir de l'extérieur et prioriser la pérennité et les retombées économiques et sociales à long terme, notamment en exigeant une politique d'essaimage chaque fois que cela est possible.

10. L'État peut valoriser et soutenir l'entrepreneurship comme il peut le restreindre, voire l'empêcher. L'État peut contribuer à créer un environnement politique, social, culturel qui attire et soutient l'entrepreneur. Il peut également, par la mise en place de divers règlements, lois et mesures, restreindre et même empêcher l'entrepreneurship.

Le discours inaugural du premier ministre Jean Charest, prononcé à Québec le 4 juin 2003, innove en cette matière. Il fait du développement de l'entrepreneurship une priorité et il propose d'améliorer la convivialité du gouvernement à l'égard des entrepreneurs. Il faut espérer que les actes suivront le discours.

Ces vérités répondent pour l'essentiel aux questions qui touchent la création de richesse et d'emplois et, en conséquence, le développement d'une localité ou d'une région. Les croyances en ces vérités sont nécessaires mais non suffisantes. Elles doivent déboucher sur de nouveaux comportements et de l'action. Il faut reconnaître aussi que les résultats ne sont pas instantanés, ce qui rend la tâche plus difficile. Voyons ensemble un scénario du comment. 
Les élus locaux ont la légitimité pour assurer directement ou par délégation le leadership de la culture entrepreneuriale dans leur milieu. Déjà, au Québec, plusieurs élus locaux assument des rôles importants en matière de développement économique, alors que d'autres attendent passivement. La démonstration de l'impact de ces deux comportements n'est plus à faire. $\mathrm{Si}$, en plus du leadership des élus locaux, on obtient la collaboration des trois acteurs suivants, la réussite devient assurée dans un délai beaucoup plus court :

- le concours des médias pour soutenir la cause et valoriser les entrepreneurs;

- l'implication de l'école pour promouvoir les attitudes et les valeurs, transmettre les connaissances et développer les compétences selon les divers niveaux;

- la participation des entrepreneurs pour témoigner de leur vécu et soutenir par divers moyens, dont la commandite, certaines activités de culture entrepreneuriale.

Progressivement, les succès obtenus renforcent les croyances dans le potentiel du milieu et produisent ce qu'on appelle en anglais l'empowerment. Voilà un mot difficilement traduisible par un seul mot français. L'empowerment origine de l'intérieur plutôt que l'inverse. Il fait appel à une autonomie de décision. Il suppose 1'accès à certaines ressources et il oblige à une imputabilité appropriée. Appliqué au développement économique local, il suggère que des leaders locaux ayant une certaine légitimité décident d'occuper plus de place en matière de développement économique. Ils décident, avec l'appui de leurs commettants, d'assumer un nouveau rôle, forçant au besoin le système externe à s'adapter.

\section{Les élus locaux ont la légitimité pour assurer directement ou par délégation le leadership de la culture entrepreneuriale dans leur milieu.}

Idéalement, les gouvernements supérieurs peuvent favoriser, voire même inciter à l'empowerment en assurant d'avance leur volonté de laisser plus de place aux instances locales en matière de développement écono- mique et en soutenant financièrement la formation ainsi que la recherche et développement.

Le discours du premier ministre du 4 juin dernier est très explicite sur la volonté de son gouvernement de confier plus de responsabilités aux élus locaux, notamment en matière de développement économique. Grâce à la formation que les unions municipales mijotent pour leurs membres, il ne fait aucun doute que ceux-ci accepteront de relever ces nouveaux défis avec enthousiasme et compétence.

\section{Conclusion}

En terminant, je veux nommer les quatre piliers de la culture entrepreneuriale proposés par la Fondation de l'entrepreneurship. Ceux-ci ont été validés pendant plusieurs années auprès de nombreuses clientèles. Les voici : l'autonomie, la responsabilité, la créativité et la solidarité. Comment peut-on se scandaliser ou s'objecter à de telles valeurs ? Quel parent, bien né, souhaite d'autres choses pour ses enfants ? Choisir la culture entrepreneuriale, c'est donner à l'ensemble du Québec un passeport vers la prospérité. C'est, ultimement, choisir d'être maîtres chez nous.

\section{Notes et références}

1 Paul-Arthur Fortin est largement connu au Québec pour son implication en entrepreneurship. Au cours des ans, plusieurs groupes ont souligné son apport à la cause. Il a cofondé la Fondation de l'entrepreneurship en 1980 et il en a dirigé les destinées jusqu'en 1997. Actuellement, il collabore avec l'équipe de Louis Jacques Filion et Danielle Luc de HEC Montréal à divers documents sur l'essaimage d'entreprises. Il participe également avec l'équipe de Gilles Saint-Pierre de l'Institut d'entrepreneuriat de l'Université de Sherbrooke et Richard Darveau du Forum francophone des affaires à la préparation d'un séminaire sur la culture entrepreneuriale pour des pays francophones de l'Afrique.

2 Fortin, Paul-A. (1986). Devenez entrepreneur, pour un Québec plus entrepreneurial, Sainte-Foy, PUL, 302 p.

3 Delavallée, Éric (2002). La culture d'entreprise pour manager autrement, Éditions d'Organisation.

4 Fortin, Paul-A. (2002). La culture entrepreneuriale, un antidote à la pauvreté, Les Éditions Transcontinental inc., Les Éditions de la Fondation de l'entrepreneurship, 248 p.

5 Global Entrepreneurship Monitor. 2002 Executive Report, Paul D. Reynolds, William D. Bygrave, Erkko Autio, Michael Hay, and the Ewing Marion Kauffman Foundation.

6 Riverin, Nathaly, «L'entrepreneuriat au Québec en 2001 », Cahier de recherche 2002-18, HEC Montréal. 